\title{
Experiments on Random Packings of Ellipsoids
}

\author{
Weining Man ${ }^{1}$, Aleksandar Donev ${ }^{4}$, Frank H. Stillinger ${ }^{2}$, Matthew T. Sullivan ${ }^{1}$, William B. Russel ${ }^{3}$, \\ David Heeger ${ }^{5}$, Souhil Inati ${ }^{5}$, \\ Salvatore Torquato ${ }^{2}$ and P. M. Chaikin ${ }^{1}$ \\ ${ }^{1}$ Dept. of Physics, ${ }^{2}$ Dept. of Chemistry, ${ }^{3}$ Dept. of Chemical Engineering \\ ${ }^{4}$ Program in Applied and Computational Mathematics \\ And Princeton Institute for the Science and Technology of Materials \\ Princeton University, Princeton NJ 08544 \\ ${ }^{5}$ Dept. of Psychology and Center for Neural Science \\ New York University, New York NY 10003
}

(Wednesday, June 08, 2005)

\begin{abstract}
Recent simulations indicate that ellipsoids can randomly pack more densely than spheres and, remarkably, for axes ratios near 1.25:1:0.8 can approach the densest crystal packing (FCC) of spheres, with packing fraction $74 \%$. We demonstrate that such dense packings are realizable. We introduce a novel way of determining packing density for a finite sample that minimizes surface effects. We have fabricated ellipsoids and show that in a sphere, the radial packing fraction, $\phi(r)$, can be obtained from $V(h)$, the volume of added fluid to fill the sphere to height $h$. We also obtain $\phi(r)$ from an MRI scan. The measurements of the overall density $\phi_{\mathrm{avr}}, \phi(r)$, and the core density $\phi_{0}=0.74 \pm 0.005$ agree with simulations.
\end{abstract}

PACS: 45.70.-n, 61.43.-j, 05.65.+b, 83.80 
The structure of liquids and glasses [1], the thermodynamics and kinetics of the crystal-liquid transition $[2,3]$, crystal nucleation and growth $[4,5,6]$, the structure and flow of granular materials $[7,8]$, the jamming transition [9], the quality of ceramics and a host of other problems in science, mathematics and technology depend on the packing properties of hard particles $[10,11,12]$. In particular, the freezing transition of simple liquids is directly related to the fact that a jammed random packing of spheres at volume fraction 0.64 [13] is less dense than the FCC crystal packing at 0.74 $[2,3,14]$. Thermodynamically, at high density, free volume considerations dominate the entropy and favor the configuration with the highest density. Until recently most studies of hard particle packings dealt with spheres. Within the past year, both experiments and simulations suggest the richness of the packing problem when the shape is modified even by the affine deformation of a sphere to an ellipsoid $[15,16]$. While the papers were inspired by random packing experiments on $M \& M \circledR$ candies [17], most of the results were based on simulations. The simulations [18] show that the density of jammed random packings of ellipsoids can approach that of the crystal packing, raising the possibility of an elusive thermodynamically stable glass.

The present study was undertaken to test whether such a dense random packing could be realized experimentally. Simulations use frictionless particles with periodic boundary conditions. Can experiments with a finite number of particles with friction yield information on the bulk packings? We show that injected fluid volume vs height in a packed spherical container provides a good measure of $\phi_{\text {bulk }}$ for rather small systems. This technique should prove useful for many granular material and packing studies. We confirm these measurements with magnetic resonance imaging (MRI) studies which provide the most direct method in the case of non-metallic particles.

In previous work, some of us have used a modified Lubachevsky-Stillinger algorithm to estimate the packing fraction of randomly jammed ellipsoids. The algorithm is an event-driven 
molecular dynamics simulation with controlled particle growth [18].The packing fraction, as a function of $\alpha$, for ellipsoids with aspect ratios $\alpha: 1: 1$ and $\alpha^{1 / 2}: 1: \alpha^{-1 / 2}$ is shown in Fig. 1a. Surprisingly the packing fraction has a cusp-like minimum at $\alpha=1$ (spheres, $\phi \approx 0.64$ ) and increases sharply with $|\alpha-1|[13]$. What was striking in these early simulations is that for ellipsoids with aspect ratios 1.25:1:0.8 the packing fraction approaches that of the densest sphere packing, which corresponds to the FCC lattice and its stacking variants at $\phi=0.7405$. Later simulations with a small periodic unit cell (32 particles/cell) and very slow growth obtained an apparently disordered packing with $\phi \approx 0.7427$, Fig. 1 insert b. Moreover, using this procedure, we found crystal ellipsoid packings that are denser than the FCC packing of spheres [16]. Confidence in the simulations was enhanced by the close agreement with the measured value of the density for M\&M candies ( $\alpha \sim 1 / 2), \phi \approx 0.71$ from simulation, $\phi=0.69$ \pm 0.01 from experiment [15].

The 1.25:1:0.8 ellipsoids used for these experiments were fabricated using a stereolithography machine 3D Systems Model SLA 250. The machine uses a UV laser with an absorption length of $100 \mu \mathrm{m}$ in monomer CIBATOLL ${ }^{\circledR}$ SL 5170. The computer controlled laser scans the surface leaving a solid polymerized layer $100 \mu \mathrm{m}$ deep. This patterned layer is then lowered and the next layer written by polymerizing the covering liquid. The beam width, which determines the xy resolution, is comparably scaled at $150 \mu \mathrm{m}$ allowing feature size of $150 \mu \mathrm{m}$ in each direction. The size of our ellipsoids was 2.344:1.875:1.5 $\mathrm{cm}$ for $<1 \%$ resolution. A picture of a few ellipsoids used in this study is shown in Figure 1 insert c.

The simplest way to measure packing fraction is to pack a container with the particles and determine the volume of fluid which fills the voids [19]. In such measurements the average packing density $\phi_{\text {avr }}$ is significantly smaller than its bulk value, $\phi_{0}$, even for large containers. This is due to the lower density in the few layers of particles near the wall (at the wall the density is zero). To minimize 
the surface to volume ratio a spherical container is preferred. We expect that for a spherical container the local density is radially symmetric. Assume that the radial density profile has the form:

$$
\phi(r)=\phi_{0}-\Delta \phi\left(\frac{R-r}{a}\right)
$$

where $a$ is the mean particle radius, $a=\left(a_{1} a_{2} a_{3}\right)^{1 / 3}$ for ellipsoids with principal axes $2 a_{1}, 2 a_{2}, 2 a_{3}$, and $\Delta \phi(\mathrm{x})$ is an approximately universal function, independent of $R$, but dependent on particle shape. The correction $\Delta \phi(x)$ decays rapidly to zero for $x>>1$. If we use (1) in $\phi_{a v r}=3 \int_{0}^{R} \phi(r) r^{2} d r / R^{3}$, we obtain the expansion of $\phi_{\mathrm{avr}}$ in powers of $\delta=a / R$ :

$$
\phi_{a v r}=\phi_{0}-3 \delta\langle\Delta \phi\rangle_{0}+6 \delta^{2}\langle\Delta \phi\rangle_{1}-3 \delta^{3}\langle\Delta \phi\rangle_{2}
$$

where $\langle\Delta \phi\rangle_{n}=\int_{0}^{R} \Delta \phi(x) x^{n} d x \approx \int_{0}^{\infty} \Delta \phi(x) x^{n} d x$. Thus, for large spherical containers the main correction to the measured total density varies as $a / R$, and a linear extrapolation of $\phi_{\text {avr }}$ vs $a / R$ to $a / R \rightarrow 0$ should give a good estimate of the bulk density $\phi_{0}[19,20]$. In Fig.2, we present the results of experiments with spherical glass marbles of diameter $1.1 \mathrm{~cm}$, mini M\&M® candies (ellipsoids of revolution or spheroids) and our fabricated ellipsoids with semi-axes $1.25 \mathrm{a}: \mathrm{a}: 0.8 \mathrm{a}, \mathrm{a}=0.9375 \mathrm{~cm}$, in spherical flasks of several sizes. The ellipsoids or spheres were poured into glass flasks which were then shaken by hand. More particles were added until further shaking failed to produce space for the addition of another particle. In order to overcome friction, we added a small amount of isopropanol as a lubricant for the marbles and ellipsoids. Also shown are computer generated jammed configurations of frictionless spheres and ellipsoids in geometrically similar spherical containers [18]. From this data, it is clear that the density is the largest for the fabricated ellipsoids and smallest for the spheres. Extrapolating the simulation results to $a / R \rightarrow 0$, we find $\phi_{0}$ (spheres) $=0.642 \pm 0.002$, $\phi_{0}\left(\mathrm{M} \& \mathrm{M}^{\prime} \mathrm{s}\right)=0.700 \pm 0.002$ and $\phi_{0}($ ellipsoids $)=0.741 \pm 0.002$. Our experimental data for glass marbles and M\&M candies agree well with simulated results of frictionless particles. However, for the 
fabricated ellipsoids, the measured packing densities are noticeably lower than the simulated result for $a / R>0.1$. Clearly a meaningful extrapolation for the $0.8: 1: 1.25$ ellipsoids to $a / R \rightarrow 0$ is difficult for the number of particles we have studied (up to $~ 1000$ particles). We need to further minimize surface effects.

The average density of a spherical core $\phi_{0}$ excluding several particle layers near the container wall should provide a very good approximation to the bulk density $\phi_{\text {bulk. }}$ We therefore devised a different experiment to allow a measurement of the radial density profile $\phi(r)$. A spherical container of radius $10 \mathrm{~cm}$ was prepared with two holes in the bottom. Tubes were attached to the holes with epoxy. The sphere was filled with particles and shaken until no further particles could be added. The sphere was then set on a digital scale and one tube was attached to a water reservoir. The other tube was attached to a pressure transducer with a sensitivity of $10^{-5}$ bar (equivalent to a water height of about $0.1 \mathrm{~mm})$. The experiment proceeded by allowing the water to slowly enter the container from the bottom while recording the weight (i.e., volume) of fluid added and the pressure (i.e. fluid level).

As we fill the sphere with a fluid from the bottom the total volume of injected liquid, $V$, as a function of the height of the liquid level $h$ (measured from the center of the flask, $-R \leq h \leq R$ ), is given by the integral:

$$
V(h)=\int_{R}^{h} 2 \pi r^{2}\left(1-\frac{h}{r}\right)[1-\phi(r)] d r .
$$

Fig. 3 shows $V(h)$ for an empty spherical container, a marbles- filled container and an ellipsoid-filled container. Equation 3 is trivially inverted to obtain $\phi(r)$ by differentiating $V(h)$ twice:

$$
1-\phi(r)=\left.\frac{-1}{2 \pi r} \frac{\partial^{2} V(h)}{\partial h^{2}}\right|_{h=r}
$$


Using Eq. (4) to obtain $\phi(r)$ from an experimental $V(h)$ is difficult. Second-order differentiation is a numerically unstable procedure. Nonetheless, averaging over intervals of $\sim 0.2 R$ we obtain $\phi(0)$ $\sim 0.64 \pm 0.05$ for marbles and $0.73 \pm 0.05$ for our ellipsoids.

It is instructive to consider the case when $\phi(r)=\phi_{0}$ is constant, which gives $V(h)$ as a simple cubic function of $h$,

$$
V(h)=\left(1-\phi_{0}\right)\left[-\frac{\pi}{3} h^{3}+\pi R^{2} h+\frac{2 \pi R^{3}}{3}\right]
$$

An important observation is that the coefficient of $h^{3}$ does not depend on $R$ and is thus independent of system size. Moreover, if $\phi(r)=\phi_{0}$, for $r<R_{l}<R, V(h)$ is a cubic function with the coefficient of the $h^{3}$ term remaining $(\pi / 3)\left(1-\phi_{0}\right)$ for a fit in the range $-R_{l}<h<R_{l}$. Therefore, a quick and rather accurate way of obtaining an estimate for the core (bulk) density $\phi_{0}$ in a packing is to fit a cubic polynomial to $V(h)$ [21]. In Fig. 3, $V(h)$ as measured is shown together with the corresponding cubic fits. For our fabricated ellipsoids we find a core density $\phi_{0}=0.739 \pm 0.005$, which is in excellent agreement with the computational result $\phi_{0=} 0.735 \pm 0.01$. For spheres we measure $\phi_{0}=0.635 \pm 0.005$, vs. the known bulk density $\phi_{0}=0.64 \pm 0.005$.

We also performed MRI scans of the ellipsoids in the same spherical container to assure that the packing did not show signs of crystal or orientational order. The space between ellipsoids was filled with water. MRI scans were collected on a Siemens 3T Allegra scanner. The images were acquired at $1 \mathrm{~mm}^{3}$ isotropic resolution per voxel using a conventional 3D FLASH sequence (acquisition matrix $=256 \times 256 \times 256, F A=10$ degree, $\mathrm{TE}=2.65 \mathrm{~ms}, \mathrm{TR}=5.5 \mathrm{~ms}, \mathrm{BW}=260 \mathrm{~Hz} / \mathrm{pixel}$ ) in a standard birdcage head coil. The manufacturer's 3D large field of view geometric distortion correction algorithm was applied to the images. Fig. 4 insert shows a gray scale slice from the MRI data and no apparent ordering is present. We can also use the MRI data for an independent measurement of 
$\phi(r)$. Simple thresholding converts the gray scale images into binary ones. Using the binary data, it is straightforward to calculate $\phi(r)$, which is shown in Fig. 4 with a solid line, while the average packing density inside a spherical core of radius $r, \phi_{a v r}(r)=\frac{3}{4 \pi r^{3}} \int_{0}^{r} \phi\left(r^{\prime}\right) 2 \pi r^{\prime 2} d r^{\prime}$, is shown as the line with circles. The oscillations come from the finite particle size and the effects of the walls. Their periodicity is approximately the mean particle diameter, $2 a, a=\left(a_{1} a_{2} a_{3}\right)^{1 / 3}$. The dashed line and triangles are results from a simulated packing with the same geometry. The average density inside the core $r<0.6 R$ is 0.735 , and the error due to finite pixel size, error in threshold and geometry distortions in the MRI scan is in total \pm 0.01 .

To summarize, we find that it is relatively straightforward to experimentally realize random ellipsoid packings whose packing density matches simulations of hard frictionless particles. We have also demonstrated a new way to measure the bulk density of an infinite random system, using a limited number of particles, which should be useful for packing measurements for other particle shapes. While we will pursue further MRI experiments which can provide more detailed information about interparticle correlations, the new technique can be used for all types of granular material whether amenable to MRI investigation or not, and is experimentally simple.

While the random packing of our 1.25:1:0.8 ellipsoids at $0.74 \pm 0.005$ is denser than random sphere packing, as dense or denser than any sphere packing, random, lattice or crystal, or any lattice packing of ellipsoids, it is not denser than the densest crystal packing of the same ellipsoids. From reference 16 , we find that the densest crystal packing of these ellipsoids is at least $\phi=0.756$.

The present paper addresses just one aspect of the broad and challenging subject concerning statistical geometry of hard-object packings. One still-unresolved question of interest involves shape dependence of the packing-fraction ratio for amorphous $v s$. crystalline arrangements of identical hard 
particles. For spheres this ratio is $\phi_{\text {amor }} / \phi_{\text {crys }} \approx 0.64 / 0.7405=0.86$, while the corresponding value for the ellipsoid shape examined herein is substantially larger: $\phi_{\text {amor }} / \phi_{\text {crys }} \approx 0.74 / 0.756=0.98$. It will be enlightening eventually to establish: (a) what particle shapes yield the attainable lower and upper limits for this ratio, (b) whether the upper limit can equal or exceed unity, and (c) whether there are useful correlations between this ratio and features of the equilibrium phase diagrams for various particle shapes.

We gratefully acknowledge experimental assistance from Vincent Pelletier and support from NSF DMR 0213706, 0243001, 0312067.and NASA NAG3-1762. 
References

1. J. D. Bernal, Nature London 183, 141 (1959), and Proc. R. Soc. London, Ser. A 280, 299 (1964)

2. P. N. Pusey, in Liquids, freezing and the glass transition, Chapter 10, Colloidal suspensions. (eds Hansen, J.P., Levesque, D. and Zinn-Justin,J.) 763-942 (Elsevier, Amsterdam, 1991).

3. P. M. Chaikin, "Thermodynamics and Hydrodynamics of Hard Spheres; the role of gravity", pp. 315-348.in Soft and Fragile Matter, Nonequilibrium Dynamics, Metastability and Flow (M. E. Cates and M. R. Evans, editors) (Institute of Physics Publishing, London, 2000)

4. B. J. Adler, and T. E. Wainwright, Phase transition for a hard sphere system. J. Chem. Phys. 27, 208-1209 (1957).

5. Z. D. Cheng, W. B. Russel, and P. M. Chaikin, Nature 401, 893 (1999)

6. M. D. Rintoul, and S. Torquato, Phys. Rev. Lett. 77, 4198 (1996)

7. H. M. Jaeger and S. E. Nagel, Science 255, 1523 (1992); H. M. Jaeger, S. E. Nagel, and R. P. Behringer, Rev. Mod. Phys. 68, 1259 (1996).

8. S. F. Edwards, Granular Matter (A. Mehta, editor) (Springer-Verlag, New York, 1994), chap. The Role of Entropy in the Specification of a Powder, pp. 121-140.

9. Liu, A. J. and Sidney R. Nagel Nature 396, 21 (1998)

10. R. Zallen, The Physics of Amorphous Solids (Wiley, New York, 1983).

11. T. Aste, D. Weaire, The Pursuit of Perfect Packing (IOP, London, 2000).

12. J. H. Conway, N. J. A. Sloane, Sphere Packings, Lattices, and Groups (Springer-Verlag, New York, ed. 3, 1999).

13. S. Torquato, T. M. Truskett, and P. G. Debenedetti, Phys. Rev. Lett. 84, 2064 (2000).

14. C. Smits, B. Van Der Most, J. K. G. Dhont and H. N. W. Lekkerkerker, Adv. Colloid Interface Sci 42, 33 (1992)

15. A. Donev, I. Cisse, D. Sachs, E. A. Variano, F. H. Stillinger, R. Connelly, S. Torquato, P. M. Chaikin, Science 303, 990, (2004).

16. A. Donev, F. H. Stillinger, P. M. Chaikin, and S. Torquato, Phys. Rev. Lett. 92, 255506 (2004)

17. M\&M'S $®$ Candies are a registered trademark of Mars, Inc.

18. "Neighbor List Collision-Driven Molecular Dynamics Simulation for Nonspherical Particles. I. Algorithmic Details II. Applications to Ellipses and Ellipsoids.", by A. Donev, F. H. Stillinger and S. Torquato, to appear in J. Comp. Phys, 2004, [physics/0110034]

19. S. Hales, Vegetable Statics., (1727) The History of Science Library, ed. Hoskin. New York: Elsevier, (1961).

20. G. D. Scott, D. M. Kilgour, J. Phys. D 2, 863 (1969).

21. Corrections for surface effects can be made by performing the cubic fit for a core region, e.g. for $V(h)$ in the range $(-R / 2)<h<(R / 2)$. If a cubic fit is used over the entire range, $-R<h<R$, we can find the error assuming a form for $\Delta \phi(x)$ in Eq. $1, \Delta \phi(x)=\beta \mathrm{e}^{-\alpha x}$, to obtain $V(h)=\mathrm{A}+\mathrm{B} h+h^{3}\left[(\pi / 3)\left(1-\phi_{0}\right)-\right.$ $35 \pi \beta /\left(2 \alpha^{3}(R / a)^{3}\right)+315 \pi \beta /\left(2 \alpha^{4}(R / a)^{4}\right)+\mathrm{O}\left((a / R)^{5}\right)$. 


\section{Figure Captions}

Fig. 1 (a) The simulated packing fraction for ellipsoids of ratios $\alpha: 1: 1$ and $\alpha^{-1 / 2}: 1: \alpha^{1 / 2}$ as a function of $\alpha$. It has a cusp-like minimum at $\alpha=1$ (spheres, $\phi=0.64$ ) and increases linearly with $|\alpha-1|$. (b) A simulated jammed configuration for 32 particles/periodic unit cell of ellipsoids with aspect ratio 1:25:1:0.8, which has a disordered packing density of 0.7427. (c) A photograph of several ellipsoids fabricated for this study.

Fig. 2 Packing fraction as a function of inverse spherical container size. Empty symbols and lines are data and extrapolation for simulated packing. The extrapolated values are: for spheres $\phi_{0}$ $=0.642 \pm 0.002$, mini M\&M's ${ }^{\circledR} \phi_{0}=0.700 \pm 0.002,1.25: 1: 0.8$ ellipsoids $\phi_{0}=0.741 \pm 0.002$. Solid symbols are the corresponding experimental results.

Fig. 3 Volume to fill a spherical container to height $h$ from center, $V(h)$, circle- empty, triangle - marble packed, star - 1.25:1:0.8 ellipsoid packed. Lines are representative of $\sim 35,000$ data points. Symbol for empty sphere is from calculation. Symbols for marbles and ellipsoids are cubic fits. Packing fractions obtained from coefficient of cubic term is equal to $\pi\left(\phi_{0}-1\right) / 3$.

Fig. 4 (a) Radial density profile $\phi(r)$. Solid line $-\phi(r)$ measured from MRI. Circles $-\phi_{\text {avr }}(r)$ measured data averaged from center out to radius $r$. Dashed line $-\phi(r)$ measured from simulations. Triangles $-\phi_{\text {avr }}(r)$ from simulations. Both measured and simulated average density in a core $r<0.6 R$ is $0.735 \pm .005$. (b) A gray scale slice from an MRI scan of the 1.25:1:0.8 ellipsoids in water in a spherical container. 


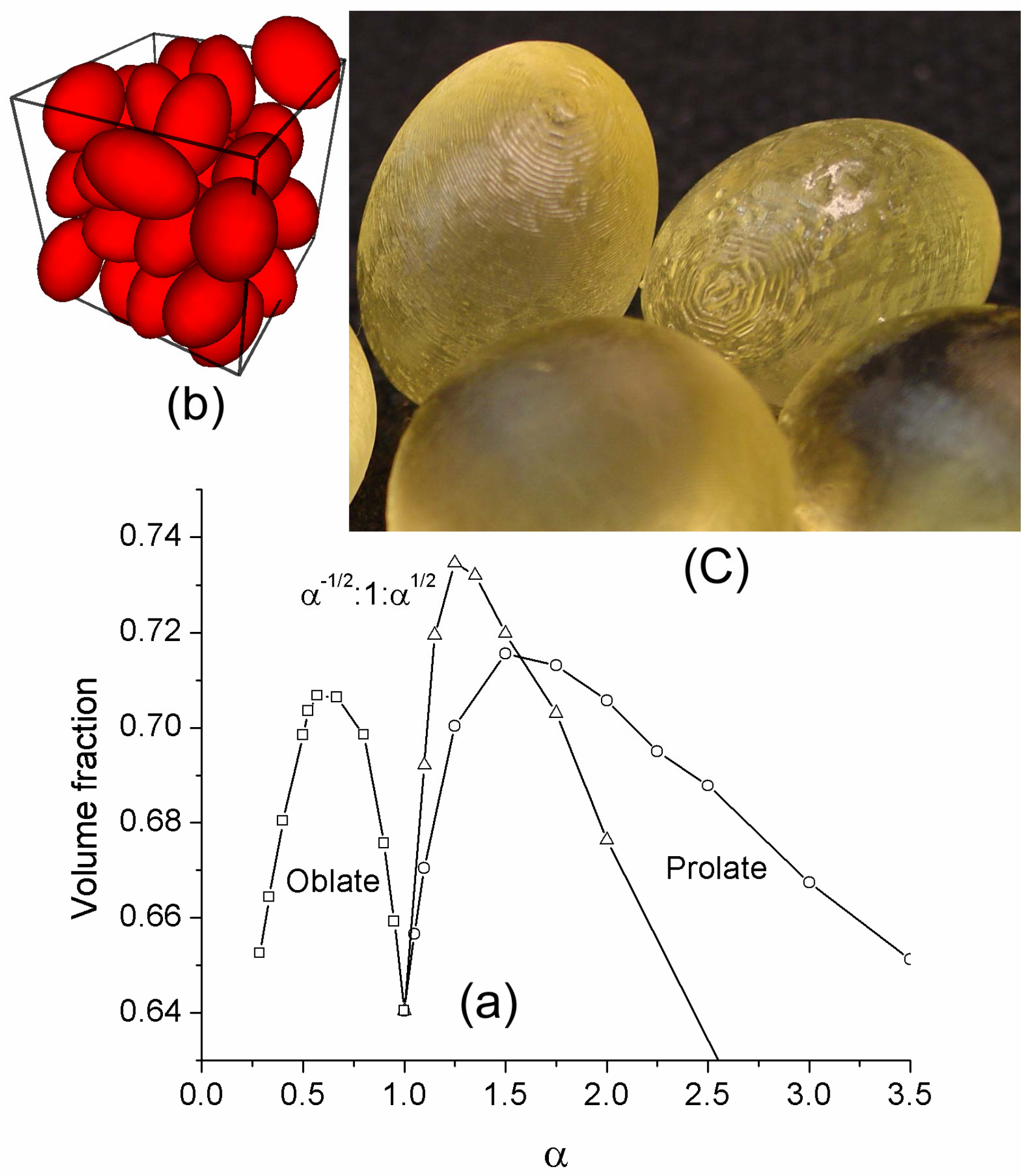




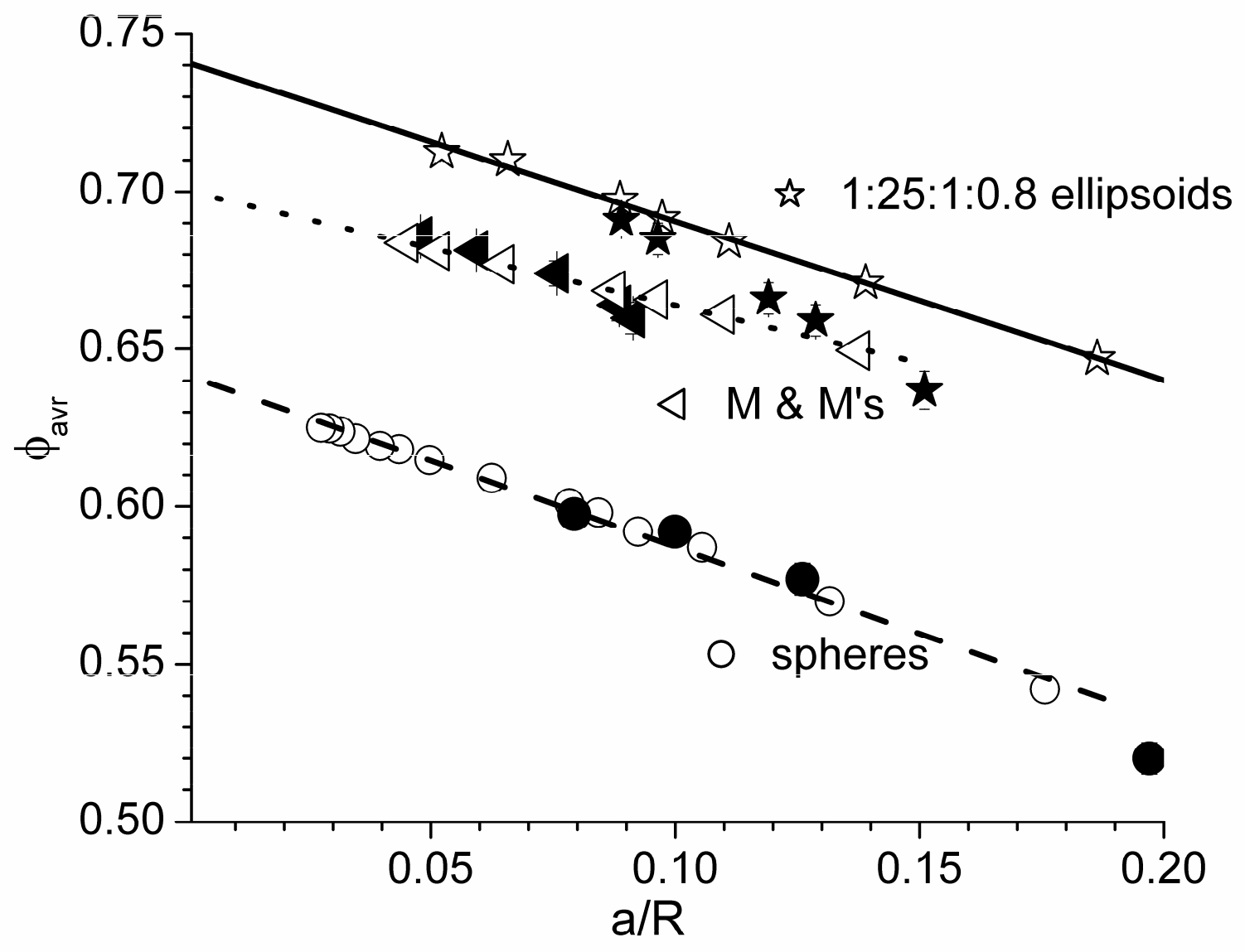




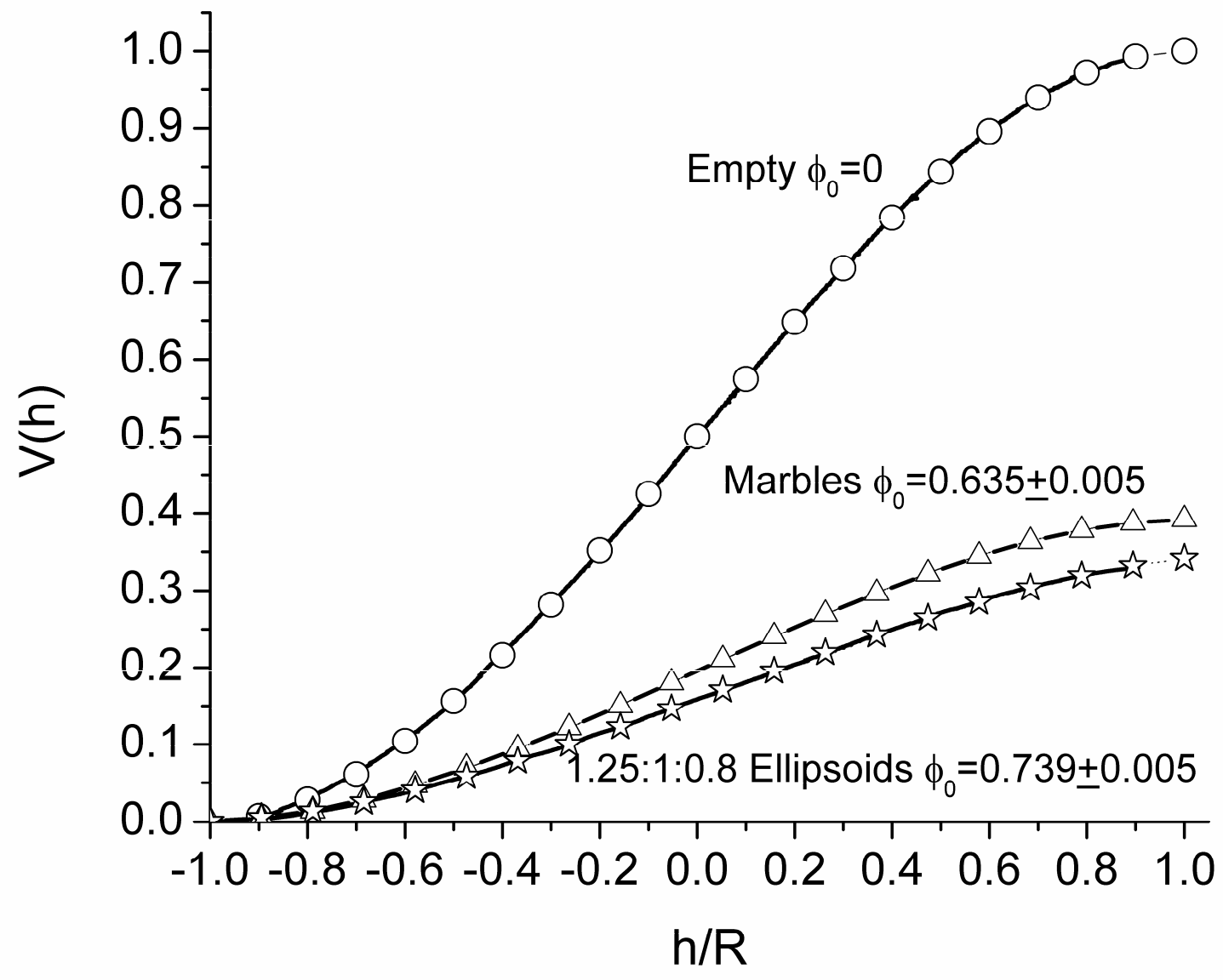




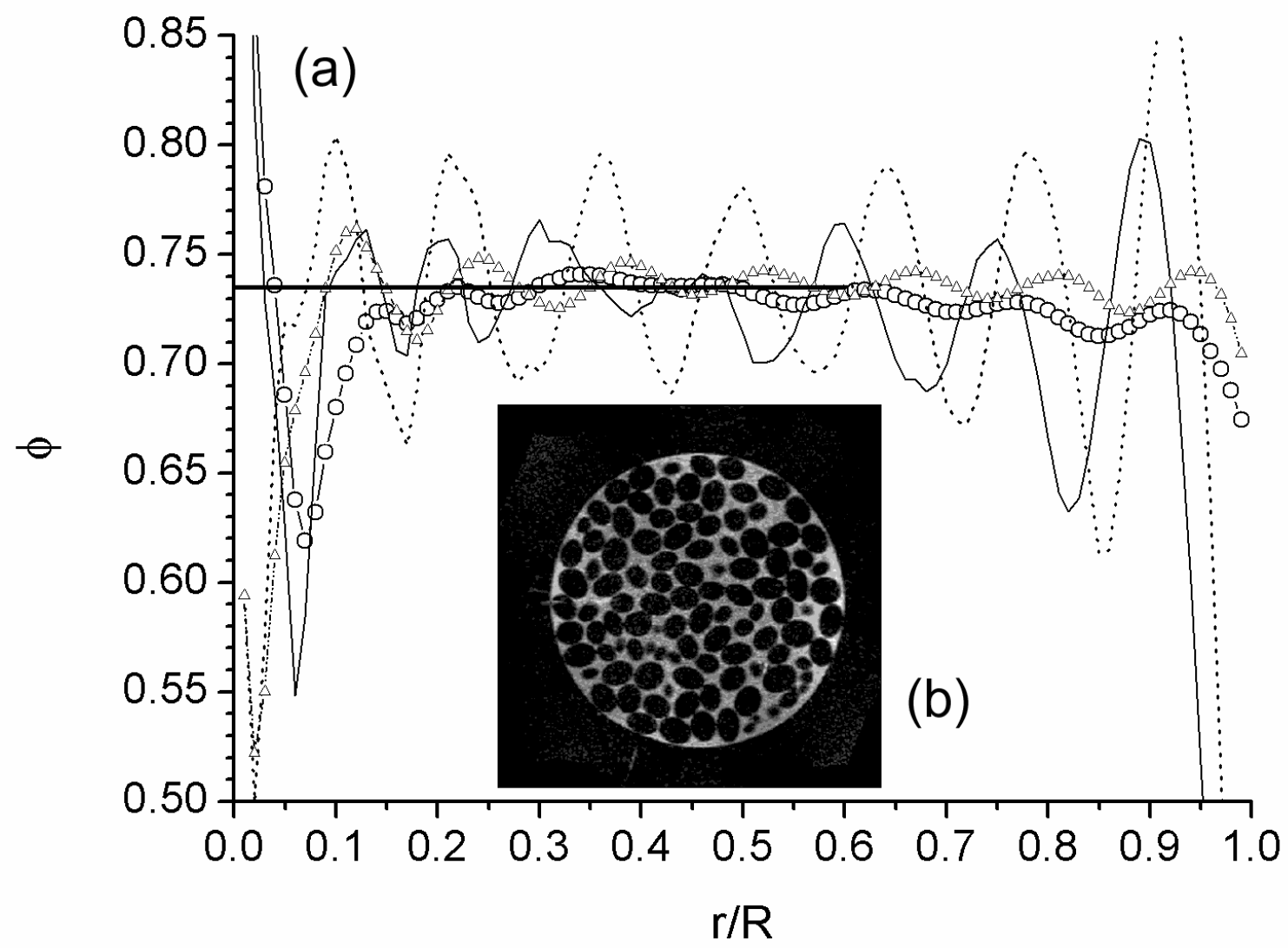

